\title{
Numerical Taxonomic Study of Some Tribes of Gramineae from Egypt
}

\author{
Ahmed Osman ${ }^{1}$, Mohammed Zaki ${ }^{2}$, Sohar Hamed ${ }^{1}$, Nagwa Hussein ${ }^{1}$ \\ ${ }^{1}$ South Valley University, Qena, Egypt; ${ }^{2}$ Cairo University, Giza, Egypt. \\ Email: ahmosman2000@yahoo.com
}

Received October 10 $0^{\text {th }}, 2010$; revised November $26^{\text {th }}, 2010$; accepted December $9^{\text {th }}, 2010$.

\begin{abstract}
A systematic study of eleven tribes of Gramineae surveyed 34 characters including fruit morphology, fruit anatomy and palynology. The results were conducted to some numerical analysis aspects. On the basis of UPGMA (Unpaired Group Method of Average) clustering and PCA (Principal component analysis), the results show congruence between the UPGMA clustering and PCA method, in suggesting two major clads/groups and five subclads.
\end{abstract}

Keywords: Gramineae, Numerical Taxonomy, UPGMA, Cladistic Tree

\section{Introduction}

Poaceae (grasses) is one of the most species-rich flowering plant families and includes many economically important crops. Parallel evolution of such features as the annual habit, $\mathrm{C} 4$ photosynthesis and several highly characteristic reproductive structures has facilitated a series of major radiations within Poaceae, culminating in the existing global distribution of about 10000 species and 700 genera [1,2]. A phylogeny of Poaceae was recently established using a combination of multiple data sets from both molecules and morphology [3], enabling improved understanding of relationships between basal and derived grasses.

Poaceae tribes and genera are subject to different studies in order to understand the phylogenetic relationships between taxa. Many attempts have been made to address phylogenetic relationships of Chloridoideae; synonym Eragrostoideae that comprises approximately 146 genera and 1360 species, whose adoption of efficient $\mathrm{C} 4$ photosynthesis had led to its successful proliferation in the tropics and subtropics [1]; mainly based on the basis of morphological and molecular data [4,5], but general agreement is still lacking. The grass tribe Triticeae includes some of the world's most important cereals and a significant number of important forage grasses [6]. Due to its renownedly complicated evolutionary history and its economic importance there has been an increasing interest in producing molecular phylogenies for the Triticeae. Attempts to unravel the relationships in the group have been based on many different types of data e.g. isozymes [7], restriction site data [8,9] and sequence data from a number of different coding and/or non-coding regions, viz.5S RNA [10]. Among the modern tools for plant taxonomy, reference [11] stated that increasing use has taken place of computers for data storage and analysis during the past twenty years. Data derived from all tools of taxonomical investigations has to be analyzed mathematically and cladistic trees have to be drawn. Despite of the criticism of using cladistic analysis in taxonomy, cladistic methods have become a most useful technical tool for clarifying intrafamilial relationships. Moreover; the advantages of using more rigorous techniques to elaborate natural classifications or evolutionary diagrams instead of those that have been used traditionally in botany have been well presented by [12] A phylogenetic analysis of Triticeae was performed by means of numerical methods due to [13]. Five methods, each based on extreme assumptions of parameters so interpreted under [14] evolutionary model, were used. The most parsimonious tree obtained served as a base for subsequent elaboration of the final tree, taking into consideration genetic information primarily, and for the erection of the proposed phylogenetic classification of Triticeae. A key is provided for identification of the groupings in the tribe. The proposed classification is discussed in the light of previous classifications, even though none of them were phylogenetic in the sense of Hennig. Reference [15] have introduced a cladistic analysis, primarily based on morphological data from 40 
taxa representing the 24 genera of the Triticeae. They used Bremer support as a measure of branch support. The trees based on morphology and on molecular data are largely incongruent. Also; [16] and [17]; in their study showed the relationships of graminid/restiid of poales in a cladistic tree. This report aims to apply numerical taxonomy; UPGMA and Cluster Analysis; to reveal better the relationships between genera within a tribe and tribes within the family based on the data collected from the previous investigations for caryopses morphology and anatomy and pollen grains morphology.

\section{Materials and Methods}

\subsection{Plant Material}

The study dealt with 34 species belonging to 25 genera of 11 tribes of Gramineae; Andropogoneae, Aristideae, Arundineae, Aveneae, Brachypodieae, Bromeae, Eragrostideae, Paniceae, Poeae, Stipeae and Triticeae. The study based on herbarial specimens dried and kept in the QNA Herbarium (in South Valley University, Qena, Botany Department) and some species received on loan from CAI Herbarium for the palynological study (Table 1). In the following analysis, species and genera constituted the OTUs (Operational Taxonomic Units). In order to broadly sample variation, the OUTs consist of a number of collections from different localities in Egypt, illustrated in Table 2.

\subsection{Characters Observations}

Table 3 shows the characters and character states scored for fruit anatomy, fruit morphology and pollen morphology, averaged for each OUT. A total of 37 characters were measured, comprising 22 qualitative and 15 quantitative characters. For recording the total characters; a main using of different microscopic techniques; light, scanning electron and stereomicroscope were used for investigating different samples and recording data collected. Table 4 shows the data matrix used for analysis of taxa studied. For some of the OTUs, some characters' observations were lacked and these omissions were coded as missing data $(-0.999)$.

\subsection{Data Analysis}

Two types of analysis were performed with STATISTICA version 5.0 computer software. Firstly, the total data coded were analyzed by the Unpired Group Method of Average (UPGMA) clustering. Construction of the tree illustrating the relationships between the studied species was performed using Arthimetic Average (UPGMA) proposed by [18]. Secondly, factor analysis and factor loadings were applied to determine the major and specific characters that aid in separation using the same program. A principal component analysis (PCA) was also performed according to [19].

\section{Results}

Figure 1 shows the UPGMA cladistic tree comprising all OTUs in the present study. The tree separated into two major clads at 100 dissimilarity distance. The first major clad at 53 dissimilarity distance, comprised only two species of the total number; Panicum turgidum and Arundo donax; while the second major clad at 93 dissimilarity comprised the rest 32 species.

The second major clad separated into two branches, the first branch includes five subclads: 1) A subclad at 86 dissimilarity distance with five species; Lamarckia aurea, Oryzopsis miliacae, Polypogon monspeliensis, Eragrostis cilinensis and Stipagrostis ciliata. 2) A subclad at 84 dissimilarity distance with Aegilops kotshyi. 3) A subclad at 80 dissimilarity distance comprises six species; Aegilops ventricosa, Hordeum murinum ssp. leporinum, Lolium perenne, Bromus scoparius, Brachypodium distachyum and Avena fatua. 4) A subclad at 74 dissimilarity distance includes nine species; Stipa capensis, Dactylis glomerata, Stipa lagascae, Bromus rubens, Echinocoloa colona, Coelachryum bervifolium, Schismus arabicus, Stipa parviflora and Aristida funiculata and 5) A subclad at 54 dissimilarity distance with nine species, Poa annua, Polypogon maritimus, Eragrostis minor, Phalaris minor, Avena barbata, Aristida mutabilis, Cenchrus ciliaris, Leptochloa fusca and Aristida adscensionis. The second branch of the second major clad comprises two species; Dactylochtenium aegyptium and Sorghum variegatum; at 66 dissimilarity distance.

Factor analysis using Principal Component Analysis (PCA) showed that the most intrinsic characters enhanced separation of the total OTUs are fruit shape, color type and fruit surface sculpture of the morphological characters, of the fruit anatomical characters; section outline shape, hull cells type, aleurone cells shape and orientation, scutellum shape and thickness and endosperm thickness are intrinsic characters for separation. Meanwhile, all the pollen characters are good data for separating of taxa; pollen class, shape, size, surface sculpture, annulus thickness, pore diameter, pollen wall thickness, sexine and nexine thickness. The characters of separation are of high factor loadings $\geq( \pm 0.7)$ Table 5 . These represented by a percentage of the total variation as $24.01 \%$ from the three factors extracted as; factor 1 is responsible for $16.54 \%$ of the variation, factor 2 is responsible for $4.34 \%$ of the variation and factor 3 is responsible for the minimum value of the total variation; is $3.13 \%$. The plot of 34 OTUs on the first two factors extracted in the PCA method is shown in (Figure 2). Plot of factor $1 / 2$ shows two groups. 1) Group of 6 . Arundo 
Table 1. List for the investigated taxa with their geographical region.

\begin{tabular}{|c|c|c|c|c|c|}
\hline No. & Species & Herb. & Collecting region & Year & Collector \\
\hline 1 & Sorghum varigatum & QNA & $\mathrm{N}$ & 2004 & A.K. OSMAN \\
\hline 2 & Aristida adscensionis & QNA & GE & 2004 & A.K. OSMAN \\
\hline 3 & Aristida funiculate & QNA & GE & 2004 & A.K. OSMAN \\
\hline 4 & Aristida mutabilis & QNA & GE & 2004 & A.K. OSMAN \\
\hline 5 & Stipagrostis ciliata & QNA & $\mathrm{R}$ & 2004 & A.K. OSMAN \\
\hline 6 & Arundo donax & QNA & $\mathrm{N}$ & 2009 & N.R.A. HUSSEIN \\
\hline 7 & Schismus arabicus & QNA & M & 2006 & A.K. OSMAN \\
\hline 8 & Avena barbata & QNA & $\mathrm{N}$ & 2005 & A.K. OSMAN \\
\hline 9 & Avena fatua & QNA & $\mathrm{N}$ & 2005 & A.K. OSMAN \\
\hline 10 & Phalaris minor & QNA & M & 2006 & A.K. OSMAN \\
\hline 11 & Polypogon maritimus & QNA & $\mathrm{N}$ & 2006 & A.K. OSMAN \\
\hline 12 & Polypogon monspeliensis & QNA & $\mathrm{N}$ & 2006,07 & A.K. OSMAN \\
\hline 13 & Brachypodium distachym & QNA & M & 2006 & A.K. OSMAN \\
\hline 14 & Bromus rubens & QNA & M & 2005 & A.K. OSMAN \\
\hline 15 & Bromus scoparius & QNA & M & 2006 & A.K. OSMAN \\
\hline 16 & Coelachyrum bervifolim & QNA & GE & 2004 & A.K. OSMAN \\
\hline 17 & Dactylochtenium aegyptium & QNA & $\mathrm{N}$ & 2006 & A.K. OSMAN \\
\hline 18 & Eragrostis cilianensis & QNA & $\mathrm{S}$ & 2005 & A.K. OSMAN \\
\hline 19 & Eragrostis minor & QNA & M & 2005 & A.K. OSMAN \\
\hline 20 & Leptochloa fusca & QNA & $\mathrm{S}$ & 2005 & A.K. OSMAN \\
\hline 21 & Cenchrus ciliaris & QNA & GE & 2004 & A.K. OSMAN \\
\hline 22 & Echinochloa colona & QNA & $\mathrm{N}$ & 2006 & A.K. OSMAN \\
\hline 23 & Panicum turgidum & QNA & $\mathrm{N}$ & 2005 & A.K. OSMAN \\
\hline 24 & Dactylis glomerata & QNA & M & 2006 & A.K. OSMAN \\
\hline 25 & Lamarckia aurea & QNA & M & 2006 & A.K. OSMAN \\
\hline 26 & Lolium perenne & QNA & $\mathrm{N}$ & 2009 & N.R.A. HUSSEIN \\
\hline 27 & Роа апnиа & QNA & $\mathrm{N}$ & 2005 & A.K. OSMAN \\
\hline 28 & Oryzopsis miliacea & CAI & M & 2006 & A.K. OSMAN \\
\hline 29 & Stipa capensis & QNA & M & 2006 & A.K. OSMAN \\
\hline 30 & Stipa lagascae & QNA & M & 2006 & A.K. OSMAN \\
\hline 31 & Stipa parviflora & QNA & M & 2006 & A.K. OSMAN \\
\hline 32 & Aegilops kotshyi & QNA & M & 2006 & A.K. OSMAN \\
\hline 33 & Aegilops ventricosa & CAI & M & 2006 & A.K. OSMAN \\
\hline 34 & Hordium murinum Subsp. Leporinum & CAI & S & 2005 & A.K. OSMAN \\
\hline
\end{tabular}

QNA = Qena Faculty of Science Herbarium (QNA a proposed Agronym); $\mathrm{M}=$ Mediterranean region; $\mathrm{N}=\mathrm{Nile}$ region; $\mathrm{R}=\mathrm{Red}$ sea coastal region; $\mathrm{S}=\mathrm{Sinai}$; $\mathrm{GE}=$ Gabel Elba. 
Table 2. List for the names of total OTUs studied and their corresponding numbers and tribe names.

\begin{tabular}{|c|c|c|c|c|c|}
\hline OTUs no. & Species name & Tribes & OTUs no. & Species name & Tribes \\
\hline Sp.1 & Sorghum varigatum & Andro & Sp.18 & Eragrostis cilianensis & Eragro \\
\hline Sp.2 & Aristida adscensionis & Arist & Sp.19 & Eragrostis minor & Eragro \\
\hline Sp.3 & Aristida funiculate & Arist & Sp.20 & Leptochloa fusca & Eragro \\
\hline Sp.4 & Aristida mutabilis & Arist & Sp.21 & Cenchrus ciliaris & Panic \\
\hline Sp.5 & Stipagrostis ciliate & Arist & Sp.22 & Echinochloa colona & Panic \\
\hline Sp.6 & Arundo donax & Arund & Sp.23 & Panicum turgidum & Panic \\
\hline Sp.7 & Schismus arabicus & Arund & Sp.24 & Dactylis glomerata & Poeae \\
\hline Sp.8 & Avena barbata & Aven & Sp.25 & Lamarckia aurea & Poeae \\
\hline Sp.9 & Avena fatua & Aven & Sp.26 & Lolium perenne & Poeae \\
\hline Sp.10 & Phalaris minor & Aven & Sp.27 & Poa annua & Poeae \\
\hline Sp.11 & Polypogon maritimus & Aven & Sp.28 & Oryzopsis miliacea & Stipeae \\
\hline Sp.12 & Polypogon monspeliensis & Aven & Sp.29 & Stipa capensis & Stipeae \\
\hline Sp.13 & Brachypodium distachym & Brach & Sp.30 & Stipa lagascae & Stipeae \\
\hline Sp.14 & Bromus rubens & Brom & Sp.31 & Stipa parviflora & Stipeae \\
\hline Sp.15 & Bromus scoparius & Brom & Sp.32 & Aegilops kotshyi & Triti \\
\hline Sp.16 & Coelachyrum bervifolim & Eragro & Sp.33 & Aegilops ventricosa & Triti \\
\hline Sp.17 & Dactylochtenium aegyptium & Eragro & Sp.34 & Hordium murinum Subsp. Leporinum & Triti \\
\hline
\end{tabular}

Andro $=$ Andropogoneae, Arist $=$ Aristideae, Arund $=$ Arundineae, Aven $=$ Aveneae, Brach $=$ Brachypodieae, Brom $=$ Bromeae, Eragro $=$ Eragrostideae, Panic $=$ Paniceae, Triti $=$ Triticeae.

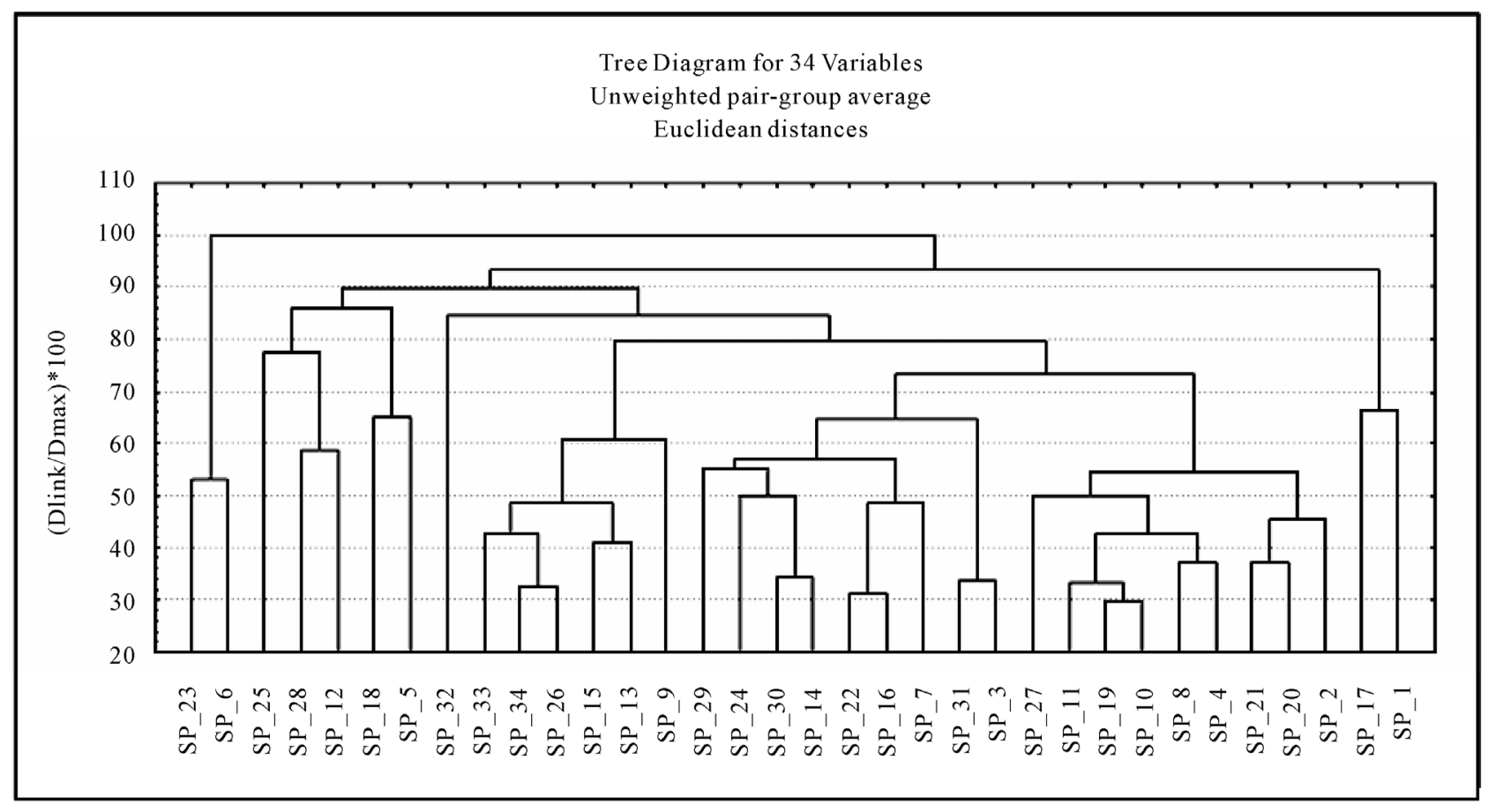

Figure 1. Cladogram of 34 species studied by UPGMA method. 
Table 3. Characters and character states used in the analysis of Gramineae tribes.

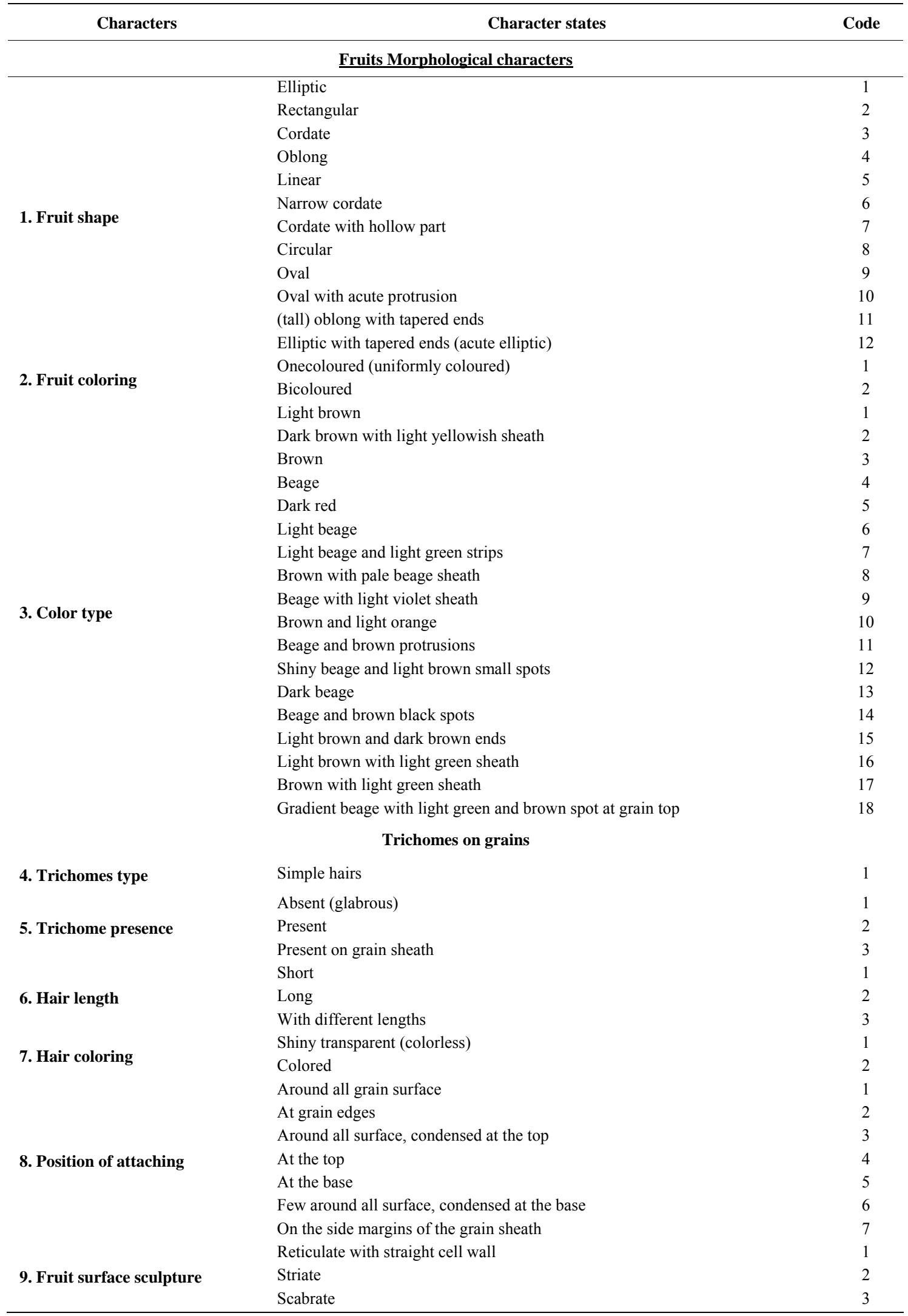




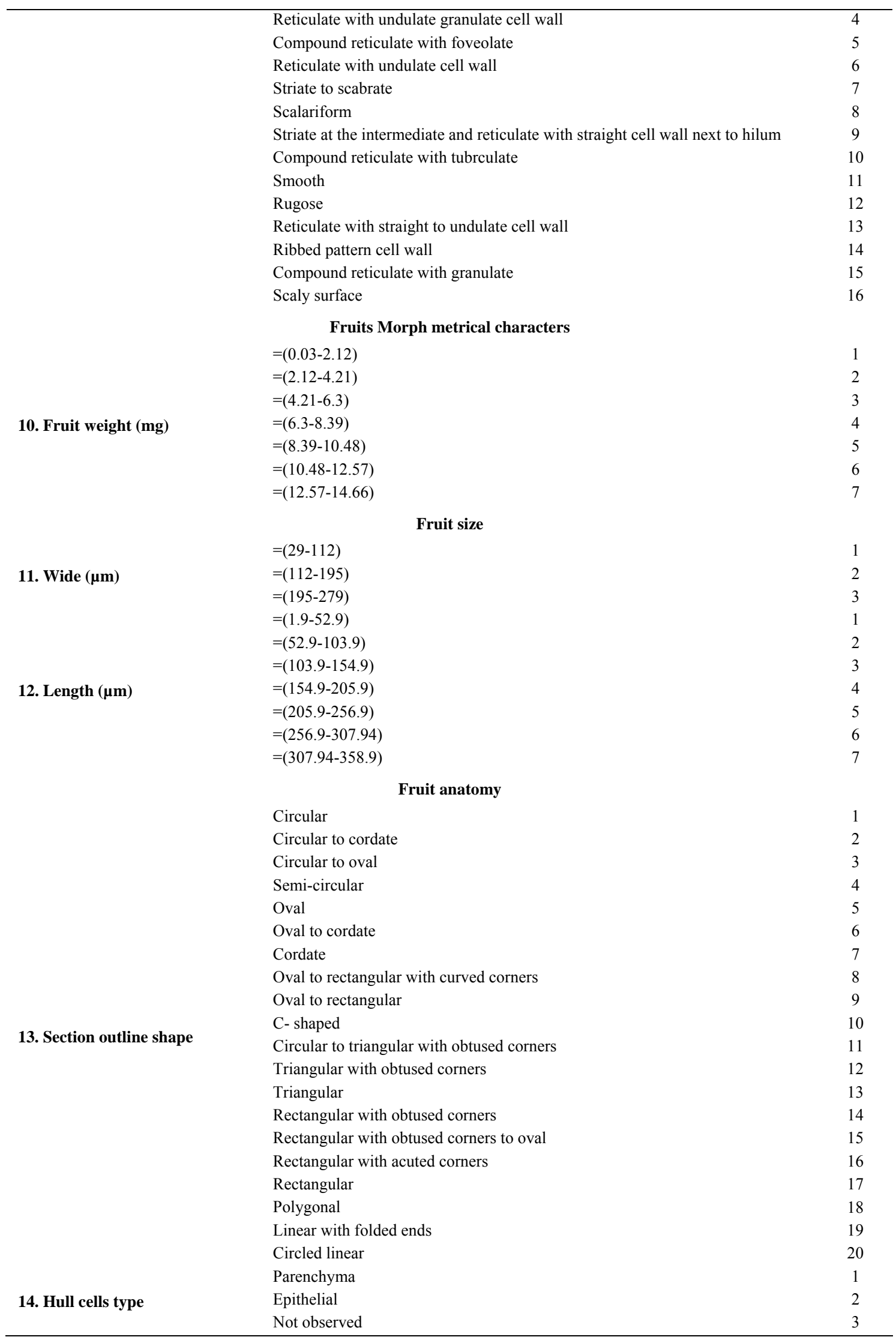




\begin{tabular}{|c|c|c|}
\hline \multirow{5}{*}{ 15. Aleurone cells shape } & Rectangular & 1 \\
\hline & Rectangular and quadrate & 2 \\
\hline & Quadrate & 3 \\
\hline & Rectangular and polygonal & 4 \\
\hline & Not observed & 5 \\
\hline \multirow{4}{*}{ 16. Aleurone cells oreintation } & Horizontal & 1 \\
\hline & Vertical & 2 \\
\hline & Horizontal and vertical & 3 \\
\hline & Not observed & 4 \\
\hline \multirow{7}{*}{ 17. Scutellum cells shape } & Strip of cells & 1 \\
\hline & Elliptic mass of cells & 2 \\
\hline & Rectangular to cordate mass of cells & 3 \\
\hline & Quinqangular mass of cells & 4 \\
\hline & Oval mass and strip of cells & 5 \\
\hline & Triangular mass and strip of cells & 6 \\
\hline & Not observed & 7 \\
\hline \multirow{2}{*}{ 18. Endosperm differentiation } & Differentiated & 1 \\
\hline & Differentiated and Undifferentiated & 2 \\
\hline \multirow{2}{*}{ 19. Type of endosperm } & Starchy & 1 \\
\hline & Starchy and fluidy & 2 \\
\hline \multirow{7}{*}{ 20. Section wide $(\mu \mathrm{m})$} & $=(5.33-31.66)$ & 1 \\
\hline & $=(31.66-57.99)$ & 2 \\
\hline & $=(57.99-84.32)$ & 3 \\
\hline & $=(84.32-110.65)$ & 4 \\
\hline & $=110.65-136.98)$ & 5 \\
\hline & $=(136.98-163.31)$ & 6 \\
\hline & $=(163.31-189.64)$ & 7 \\
\hline \multirow{7}{*}{ 21. Section length $(\mu \mathrm{m})$} & $=(33.33-71.94)$ & 1 \\
\hline & $=(71.94-110.55)$ & 2 \\
\hline & $=(110.55-149.16)$ & 3 \\
\hline & $=(149.16-187.77)$ & 4 \\
\hline & $=(187.77-226.38)$ & 5 \\
\hline & $=(226.38-264.99)$ & 6 \\
\hline & $=(264.99-303.6)$ & 7 \\
\hline \multirow{6}{*}{ 22. Hull cells thickness $(\mu \mathrm{m})$} & Not ovserved & 0 \\
\hline & $=(9.39-14.79)$ & 1 \\
\hline & $=(14.79-20.19)$ & 2 \\
\hline & $=(20.19-25.59)$ & 3 \\
\hline & $=(25.59-30.99)$ & 4 \\
\hline & $=(30.99-36.39)$ & 5 \\
\hline \multirow{6}{*}{ 23. Seed coat thickness $(\mu \mathrm{m})$} & $=(2.43-8.63)$ & 1 \\
\hline & $=(8.63-14.83)$ & 2 \\
\hline & $=(14.83-21.03)$ & 3 \\
\hline & $=(21.03-27.23)$ & 4 \\
\hline & $=(27.23-33.43)$ & 5 \\
\hline & $=(33.43-39.63)$ & 6 \\
\hline \multirow{7}{*}{$\begin{array}{l}\text { 24. Aleurone layer thickness } \\
(\mu \mathrm{m})\end{array}$} & Not observed & 0 \\
\hline & $=(1.92-9.42)$ & 1 \\
\hline & $=(9.42-16.92)$ & 2 \\
\hline & $=(19.92-24.42)$ & 3 \\
\hline & $=(24.42-31.92)$ & 4 \\
\hline & $=(31.92-39.42)$ & 5 \\
\hline & $=(39.42-46.92)$ & 6 \\
\hline \multirow{4}{*}{ 25. Scutellum thickness ( $\mu \mathrm{m})$} & Not observed & 0 \\
\hline & $=(5.54-85.94)$ & 1 \\
\hline & $=(85.94-166.43)$ & 2 \\
\hline & $=(166.34-246.74)$ & 3 \\
\hline
\end{tabular}




\begin{tabular}{|c|c|c|}
\hline & $=(246.74-327.14)$ & 4 \\
\hline & $=(327.14-407.54)$ & 5 \\
\hline & $=(407.54-487.94)$ & 6 \\
\hline \multirow{9}{*}{ 26. Endosperm thickness $(\mu \mathrm{m})$} & Thicknesses in the range codes $1 \& 4$ & 7 \\
\hline & Thicknesses in the range codes $1 \& 3$ & 8 \\
\hline & $=(21.52-125.02)$ & 1 \\
\hline & $=(125.02-228.52)$ & 2 \\
\hline & $=(228.52-332.02)$ & 3 \\
\hline & $=(332.02-435.52)$ & 4 \\
\hline & $=(435.52-539.02)$ & 5 \\
\hline & $=(539.02-642.52)$ & 6 \\
\hline & Two different thicknesses in range of codes $1 \& 2$ & 7 \\
\hline \multicolumn{3}{|c|}{ Pollen grains Morphological characters } \\
\hline \multirow{2}{*}{ 27. Pollen class } & Monoporate & 1 \\
\hline & Diporate & 2 \\
\hline 28. Annulus & Pollen annulate & 1 \\
\hline 29. Operculum & Pollen operculate & 1 \\
\hline \multirow{3}{*}{ 30. Pollen size } & Pollen small & 1 \\
\hline & Pollen medium & 2 \\
\hline & Pollen Oblate-spheroidal & 1 \\
\hline \multirow{3}{*}{ 31. Pollen shape } & Pollen Suboblate & 2 \\
\hline & Pollen Prolate-spheroidal & 3 \\
\hline & Pollen spheroidal & 4 \\
\hline \multirow{4}{*}{ 32. Surface sculpturing } & Areolate & 1 \\
\hline & Granulate & 2 \\
\hline & Scabrate & 3 \\
\hline & verrucate & 4 \\
\hline \multicolumn{3}{|c|}{ Pollen grains Morph metrical characters } \\
\hline \multirow{6}{*}{ 33. Annulus thickness ( $\mu \mathrm{m})$} & $=(1.16-1.52)$ & 1 \\
\hline & $=(1.52-1.88)$ & 2 \\
\hline & $=(1.88-2.24)$ & 3 \\
\hline & $=(2.24-2.6)$ & 4 \\
\hline & $=(2.6-2.96)$ & 5 \\
\hline & $=(2.96-3.32)$ & 6 \\
\hline \multirow{5}{*}{ 34. Pore diameter $(\mu \mathrm{m})$} & $=(1.57-2.17)$ & 1 \\
\hline & $=(2.17-2.77)$ & 2 \\
\hline & $=(2.77-3.37)$ & 3 \\
\hline & $=(3.37-3.97)$ & 4 \\
\hline & $=(3.97-4.57)$ & 5 \\
\hline \multirow{5}{*}{ 35. Pollen wall thickness $(\mu \mathrm{m})$} & $=(0.698-0.848)$ & 1 \\
\hline & $=(0.848-0.998)$ & 2 \\
\hline & $=(0.998-1.148)$ & 3 \\
\hline & $=(1.148-1.298)$ & 4 \\
\hline & $=(1.298-1.448)$ & 5 \\
\hline \multirow{6}{*}{ 36. Sexine thickness $(\mu \mathrm{m})$} & $=(0.434-0.454)$ & 1 \\
\hline & $=(0.454-0.474)$ & 2 \\
\hline & $=(0.474-0.494)$ & 3 \\
\hline & $=(0.494-0.514)$ & 4 \\
\hline & $=(0.514-0.534)$ & 5 \\
\hline & $=(0.534-0.554)$ & 6 \\
\hline \multirow{6}{*}{ 37. Nexine thickness $(\mu \mathrm{m})$} & $=(0.314-0.354)$ & 1 \\
\hline & $=(0.354-0.394)$ & 2 \\
\hline & $=(0.394-0.434)$ & 3 \\
\hline & $=(0.434-0.474)$ & 4 \\
\hline & $=(0.474-0.514)$ & 5 \\
\hline & $=(0.514-0.554)$ & 6 \\
\hline
\end{tabular}


Table 4. Data matrix used in the numerical analysis for some Gramineae tribes.

\begin{tabular}{|c|c|c|c|c|c|c|c|c|c|c|c|c|c|c|c|c|}
\hline & Sp.1 & Sp.2 & Sp.3 & Sp.4 & Sp.5 & Sp.6 & Sp.7 & Sp.8 & Sp.9 & Sp.10 & Sp.11 & Sp.12 & Sp.13 & Sp.14 & Sp.15 & Sp.16 \\
\hline 1 & 3 & 5 & 5 & 5 & 5 & -0.999 & 9 & 4 & 4 & 3 & 3 & 4 & 5 & 11 & 5 & 7 \\
\hline 2 & 2 & 1 & 1 & 1 & 1 & -0.999 & 2 & 1 & 1 & 1 & 1 & 1 & 1 & 1 & 1 & 1 \\
\hline 3 & 18 & 9 & 8 & 3 & 1 & -0.999 & 12 & 6 & 4 & 3 & 1 & 16 & 5 & 5 & 5 & 5 \\
\hline 4 & 0 & 1 & 1 & 1 & 1 & -0.999 & 0 & 1 & 1 & 1 & 0 & 0 & 1 & 1 & 1 & 0 \\
\hline 5 & 0 & 1 & 1 & 1 & 1 & -0.999 & 0 & 1 & 2 & 1 & 0 & 0 & 1 & 1 & 1 & 0 \\
\hline 6 & 0 & 2 & 2 & 2 & 2 & -0.999 & 0 & 2 & 2 & 1 & 0 & 0 & 1 & 1 & 1 & 0 \\
\hline 7 & 0 & 5 & 5 & 5 & 5 & -0.999 & 0 & 3 & 1 & 1 & 0 & 0 & 2 & 1 & 7 & 0 \\
\hline 8 & 2 & 7 & 6 & 2 & 14 & -0.999 & 11 & 2 & 2 & 3 & 7 & 12 & 2 & 15 & 2 & 6 \\
\hline 9 & 1 & 1 & 1 & 1 & 1 & -0.999 & 1 & 1 & 4 & 1 & 1 & 1 & 2 & 2 & 1 & 1 \\
\hline 10 & 3 & 3 & 1 & 1 & 1 & -0.999 & 1 & 2 & 5 & 3 & 1 & 1 & 2 & 3 & 1 & 2 \\
\hline 11 & 1 & 3 & 1 & 1 & 1 & -0.999 & 1 & 1 & 4 & 1 & 1 & 1 & 1 & 1 & 2 & 1 \\
\hline 12 & 16 & 5 & 20 & 4 & 1 & -0.999 & 12 & 6 & 17 & 5 & 1 & 2 & 10 & 15 & 10 & 10 \\
\hline 13 & 1 & 1 & 3 & 2 & 2 & -0.999 & 3 & 3 & 3 & 3 & 3 & 1 & 3 & 3 & 3 & 1 \\
\hline 14 & 1 & 1 & 5 & 1 & 1 & -0.999 & 1 & 1 & 1 & 4 & 2 & 1 & 1 & 2 & 1 & 1 \\
\hline 15 & 1 & 1 & 4 & 3 & 1 & -0.999 & 1 & 2 & 2 & 2 & 2 & 2 & 1 & 2 & 2 & 1 \\
\hline 16 & 2 & 2 & 7 & 2 & 1 & -0.999 & 1 & 4 & 1 & 1 & 1 & 7 & 7 & 7 & 7 & 1 \\
\hline 17 & 1 & 1 & 1 & 1 & 1 & -0.999 & 1 & 1 & 1 & 1 & 1 & 1 & 2 & 1 & 1 & 1 \\
\hline 18 & 1 & 1 & 2 & 1 & 1 & -0.999 & 1 & 1 & 1 & 1 & 1 & 1 & 1 & 1 & 1 & 1 \\
\hline 19 & 2 & 1 & 2 & 2 & 2 & -0.999 & 2 & 4 & 4 & 2 & 2 & 1 & 2 & 2 & 1 & 2 \\
\hline 20 & 3 & 1 & 1 & 1 & 1 & -0.999 & 1 & 3 & 5 & 2 & 1 & 1 & 3 & 2 & 2 & 2 \\
\hline 21 & 1 & 1 & 0 & 1 & 1 & -0.999 & 0 & 0 & 0 & 0 & 0 & 2 & 0 & 0 & 0 & 3 \\
\hline 22 & 2 & 1 & 1 & 1 & 1 & -0.999 & 2 & 1 & 4 & 2 & 1 & 1 & 2 & 1 & 2 & 2 \\
\hline 23 & 1 & 1 & 0 & 1 & 1 & -0.999 & 1 & 5 & 5 & 3 & 2 & 2 & 1 & 3 & 3 & 1 \\
\hline 24 & 2 & 2 & 0 & 2 & 1 & -0.999 & 1 & 5 & 1 & 1 & 1 & 0 & 0 & 0 & 0 & 1 \\
\hline 25 & 1 & 1 & 1 & 2 & 4 & -0.999 & 3 & 5 & 6 & 4 & 4 & 2 & 7 & 2 & 1 & 1 \\
\hline 26 & 1 & -0.999 & -0.999 & -0.999 & 1 & 1 & -0.999 & -0.999 & 1 & -0.999 & -0.999 & -0.999 & 2 & -0.999 & 2 & -0.999 \\
\hline 27 & 2 & -0.999 & -0.999 & -0.999 & 1 & 2 & -0.999 & -0.999 & 2 & -0.999 & -0.999 & -0.999 & 2 & -0.999 & 2 & -0.999 \\
\hline 28 & 2 & -0.999 & -0.999 & -0.999 & 4 & 1 & -0.999 & -0.999 & 1 & -0.999 & -0.999 & -0.999 & 4 & -0.999 & 4 & -0.999 \\
\hline 29 & 3 & -0.999 & -0.999 & -0.999 & 2 & 1 & -0.999 & -0.999 & 3 & -0.999 & -0.999 & -0.999 & 2 & -0.999 & 2 & -0.999 \\
\hline 30 & 2 & -0.999 & -0.999 & -0.999 & 3 & 2 & -0.999 & -0.999 & 3 & -0.999 & -0.999 & -0.999 & 1 & -0.999 & 4 & -0.999 \\
\hline 31 & 1 & -0.999 & -0.999 & -0.999 & 1 & 2 & -0.999 & -0.999 & 4 & -0.999 & -0.999 & -0.999 & 2 & -0.999 & 2 & -0.999 \\
\hline 32 & 5 & -0.999 & -0.999 & -0.999 & 1 & 1 & -0.999 & -0.999 & 1 & -0.999 & -0.999 & -0.999 & 1 & -0.999 & 2 & -0.999 \\
\hline 33 & 2 & -0.999 & -0.999 & -0.999 & 1 & 2 & -0.999 & -0.999 & 1 & -0.999 & -0.999 & -0.999 & 2 & -0.999 & 2 & -0.999 \\
\hline 34 & 3 & -0.999 & -0.999 & -0.999 & 4 & 3 & -0.999 & -0.999 & 3 & -0.999 & -0.999 & -0.999 & 2 & -0.999 & 3 & -0.999 \\
\hline
\end{tabular}




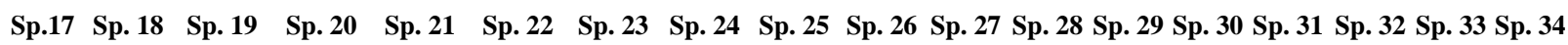

\begin{tabular}{|c|c|c|c|c|c|c|c|c|c|c|c|c|c|c|c|c|c|c|}
\hline 1 & 9 & 8 & 1 & 1 & 3 & 3 & 6 & 10 & 12 & 2 & 1 & 9 & 4 & 5 & 5 & 4 & 2 & 4 \\
\hline 2 & 2 & 1 & 1 & 2 & 2 & 2 & 1 & 1 & 2 & 1 & 1 & 1 & 1 & 1 & 1 & 1 & 1 & 1 \\
\hline 3 & 11 & 3 & 1 & 10 & 14 & 7 & 4 & 3 & 15 & 1 & 3 & 13 & 1 & 3 & 4 & 17 & 1 & 4 \\
\hline 4 & 0 & 0 & 0 & 0 & 0 & 0 & 0 & 0 & 1 & 0 & 1 & 1 & 2 & 1 & 1 & 1 & 1 & 0 \\
\hline 5 & 0 & 0 & 0 & 0 & 0 & 0 & 0 & 0 & 2 & 0 & 1 & 1 & 3 & 1 & 1 & 1 & 1 & 0 \\
\hline 6 & 0 & 0 & 0 & 0 & 0 & 0 & 0 & 0 & 2 & 0 & 2 & 2 & 2 & 2 & 1 & 2 & 2 & 0 \\
\hline 7 & 0 & 0 & 0 & 0 & 0 & 0 & 0 & 0 & 5 & 0 & 4 & 4 & 6 & 1 & 5 & 4 & 4 & 0 \\
\hline 8 & 10 & 16 & 1 & 1 & 6 & 5 & 1 & 6 & 6 & 2 & 8 & 12 & 9 & 13 & 2 & 2 & 2 & 4 \\
\hline 9 & 1 & 1 & 1 & 1 & 1 & 1 & 1 & 1 & 1 & 2 & 1 & 1 & 1 & 1 & 2 & 3 & 5 & 2 \\
\hline 10 & 1 & 1 & 1 & 1 & 2 & 3 & 5 & 1 & 2 & 3 & 1 & 2 & 1 & 1 & 2 & 4 & 5 & 2 \\
\hline 11 & 1 & 1 & 1 & 1 & 1 & 1 & 1 & 1 & 1 & 1 & 1 & 1 & 1 & 1 & 5 & 1 & 1 & 1 \\
\hline 12 & 18 & 1 & 2 & 1 & 5 & 13 & -0.999 & 11 & -0.999 & 8 & 3 & 1 & 14 & 14 & 19 & 7 & 9 & 8 \\
\hline 13 & 3 & 3 & 3 & 3 & 3 & 1 & -0.999 & 3 & -0.999 & 3 & 3 & 3 & 3 & 3 & 3 & 3 & 3 & 3 \\
\hline 14 & 1 & 1 & 5 & 1 & 1 & 1 & -0.999 & 5 & -0.999 & 1 & 3 & 3 & 1 & 2 & 5 & 1 & 2 & 1 \\
\hline 15 & 1 & 1 & 4 & 1 & 1 & 1 & -0.999 & 4 & -0.999 & 2 & 1 & 1 & 1 & 1 & 4 & 1 & 2 & 2 \\
\hline 16 & 6 & 5 & 1 & 1 & 1 & 1 & -0.999 & 7 & -0.999 & 7 & 7 & 7 & 1 & 7 & 7 & 3 & 7 & 7 \\
\hline 17 & 1 & 1 & 1 & 1 & 1 & 1 & -0.999 & 1 & -0.999 & 1 & 1 & 1 & 1 & 1 & 1 & 1 & 1 & 1 \\
\hline 18 & 1 & 1 & 1 & 1 & 1 & 1 & -0.999 & 1 & -0.999 & 1 & 1 & 1 & 1 & 1 & 1 & 1 & 1 & 1 \\
\hline 19 & 2 & 2 & 2 & 1 & 2 & 2 & -0.999 & 3 & -0.999 & 3 & 2 & 2 & 1 & 2 & 1 & 6 & 5 & 6 \\
\hline 20 & 1 & 1 & 1 & 1 & 3 & 1 & -0.999 & 1 & -0.999 & 3 & 1 & 1 & 1 & 1 & 2 & 5 & 6 & 4 \\
\hline 21 & 0 & 0 & 0 & 0 & 0 & 5 & -0.999 & 0 & -0.999 & 0 & 0 & 0 & 0 & 0 & 0 & 0 & 0 & 0 \\
\hline 22 & 2 & 1 & 2 & 1 & 1 & 1 & -0.999 & 1 & -0.999 & 4 & 6 & 6 & 1 & 2 & 2 & 3 & 6 & 5 \\
\hline 23 & 1 & 1 & 0 & 1 & 1 & 2 & -0.999 & 0 & -0.999 & 5 & 2 & 2 & 1 & 1 & 0 & 5 & 4 & 6 \\
\hline 24 & 7 & 8 & 1 & 1 & 1 & 1 & -0.999 & 0 & -0.999 & 0 & 0 & 0 & 1 & 0 & 0 & 6 & 0 & 0 \\
\hline 25 & 2 & 3 & 4 & 3 & 4 & 4 & -0.999 & 4 & -0.999 & 3 & 3 & 3 & 5 & 3 & 1 & 5 & 6 & 3 \\
\hline 26 & 2 & -0.999 & -0.999 & -0.999 & -0.999 & -0.999 & 1 & -0.999 & -0.999 & 1 & -0.999 & 1 & -0.99 & -0.999 & 0.999 & -0.999 & 2 & 2 \\
\hline 27 & 2 & -0.999 & -0.999 & -0.999 & -0.999 & -0.999 & 2 & -0.999 & -0.999 & 2 & -0.999 & 2 & -0.99 & -0.999 & 0.999 & -0.999 & 2 & 2 \\
\hline 28 & 3 & -0.999 & -0.999 & -0.999 & -0.999 & -0.999 & 1 & -0.999 & -0.999 & 4 & -0.999 & 1 & -0.99 & -0.999 & 0.999 & -0.999 & 4 & 1 \\
\hline 29 & 2 & -0.999 & -0.999 & -0.999 & -0.999 & -0.999 & 1 & -0.999 & -0.999 & 4 & -0.999 & 2 & -0.99 & -0.999 & -0.999 & -0.999 & 1 & 2 \\
\hline 30 & 2 & -0.999 & -0.999 & -0.999 & -0.999 & -0.999 & 4 & -0.999 & -0.999 & 3 & -0.999 & 1 & -0.99 & -0.999 & 0.999 & -0.999 & 5 & 3 \\
\hline 31 & 1 & -0.999 & -0.999 & -0.999 & -0.999 & -0.999 & 1 & -0.999 & -0.999 & 4 & -0.999 & 2 & -0.99 & -0.999 & 0.999 & -0.999 & 3 & 2 \\
\hline 32 & 3 & -0.999 & -0.999 & -0.999 & -0.999 & -0.999 & 2 & -0.999 & -0.999 & 3 & -0.999 & 2 & -0.99 & -0.999 & 0.999 & -0.999 & 4 & 1 \\
\hline 33 & 2 & -0.999 & -0.999 & -0.999 & -0.999 & -0.999 & 2 & -0.999 & -0.999 & 2 & -0.999 & 2 & -0.99 & -0.999 & -0.999 & -0.999 & 2 & 2 \\
\hline 34 & 3 & -0.999 & -0.999 & -0.999 & -0.999 & -0.999 & 1 & -0.999 & -0.999 & 3 & -0.999 & 3 & -0.99 & -0.999 & -0.999 & -0.999 & 3 & 2 \\
\hline
\end{tabular}




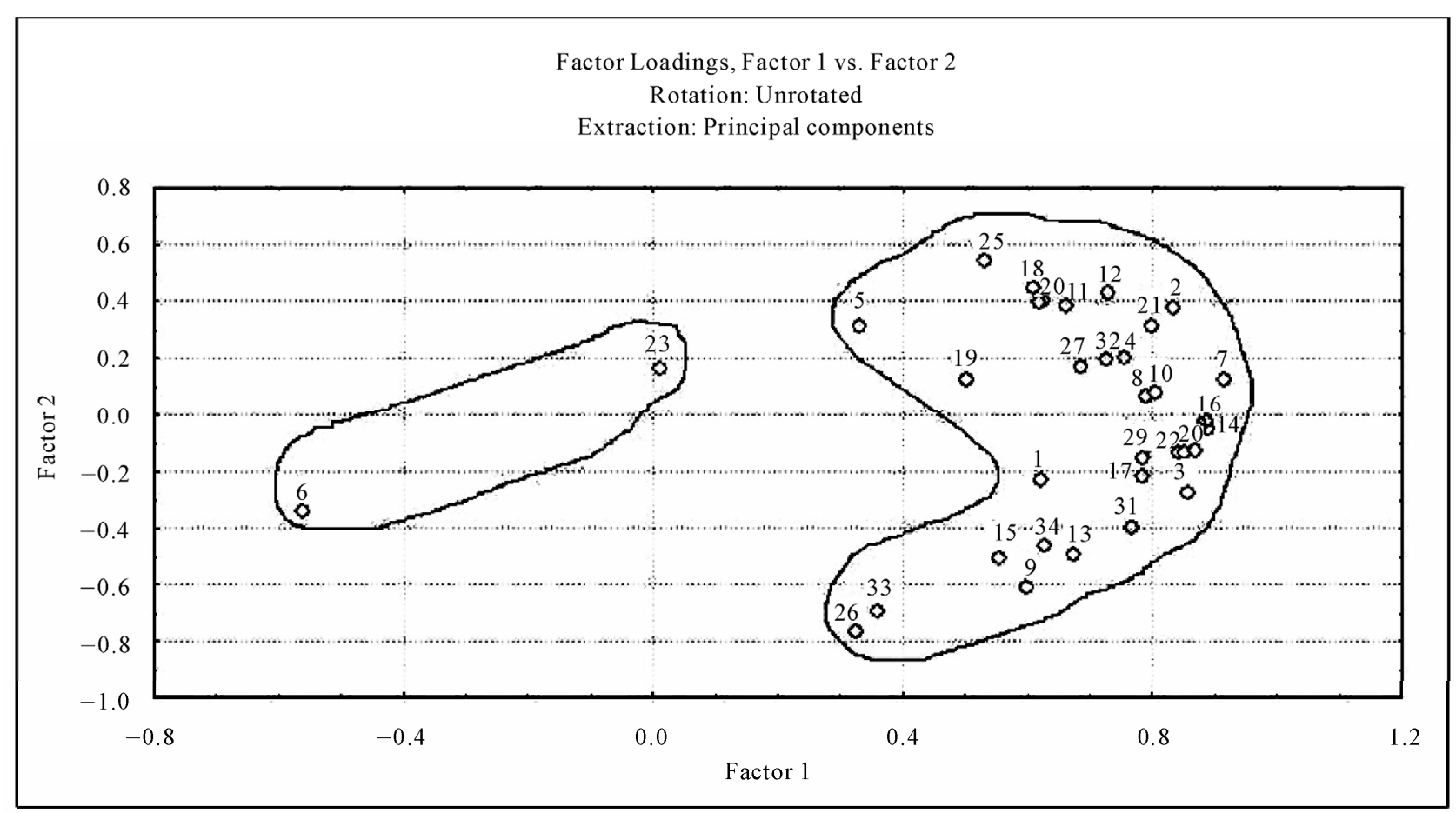

Figure 2. Scatter-plot of 34 studied taxa plotted against the first factor by the second factor.

donax and 23. Panicum turgidum. 2) Group including the rest 32 species. There are some characters; character of trichomes type, annulus of the pollen and also the operculum of the pollen grain (Table 3); which not been fitted into the data matrix because they are of only one code, so they were excluded in the analysis because they had no variation in the matrix.

\section{Discussion}

In the present study a large number of grains macro- and micro-morphological, anatomical and pollen grains characters were scored and numerical methods (UPGMA and PCA) were applied to study the relationship among eleven Poaceae tribes and estimate the level of variation within and among these tribes. UPGMA gives insight into degree of similarity among the studied species and whether they form groups // clusters and gives an indication of the level of variation within and between tribes. PCA reflects which characters are important on the axes, and indicates the significant characters based on the highest factor score (Table 5). Therefore it becomes clear which characters cause the separation between groups and can be useful to distinguish taxa. Pollen grains showed the most powerful significant characters, whereas all characters have been recorded are of high factor scores. Generally, our results show congruence between the UPGMA clustering and PCA analysis in suggesting two main groups and five subgroups which included the distribution of eleven tribes studied.

Our UPGMA results show that the tribe Andropogoneae is separated in one branch of the cladistic tree, the tribe Aristidieae is separated in three branches of the tree through three different subclads // subgroups. The tribe Arundineae with two species is separated in two branches of different clads in the tree. The tribe Aveneae is separated in five branches of the tree while the tribe Brachypodieae is separated in one branch and the Bromeae is separated in two branches within two different subclads. The Eragrostideae separated in four branches and the Paniceae separated in three branches. The Stipeae separated in four branches in only two clads while the Triticeae separated in three branches in also two clads. All the mentioned species, tribes, major clades and subclads are arranged as the following:

The first main clads // groups of two species 6. Arundo donax (Tribe: Arundineae) and 23. Panicum turgidum (Tribe: Paniceae). While the second main group includes a large variety of taxa from different tribes; 32 species of tribes: Andropogoneae, Aristideae, Arundineae, Aveneae, Brachypodieae, Bromeae, Eragrostideae, Paniceae, Stipeae and Triticeae. These tribes are separated through five distinct subgroups: 1) Sub-clad of species 25. Lamarckia aurea, 28. Oryzopsis miliacea, 12. Polypogon monspeliensis, 18. Eragrostis cilianensis and 5. Stipagrostis ciliata belonging to Poeae, Stipeae, Aveneae, Eragrostideae, and Aristideae. 2) Sub-clad only of species 
Table 5. Factor loadings showed the most intrinsic characters enhanced separations of the studied species.

\begin{tabular}{|c|c|c|c|c|}
\hline \multirow{2}{*}{\multicolumn{2}{|c|}{ Characters }} & \multicolumn{3}{|c|}{$\begin{array}{c}\text { Factor Loadings } \\
\text { Rotation: Un-rotated } \\
\text { Extraction: Principal components }\end{array}$} \\
\hline & & Factor 1 & Factor 2 & Factor 3 \\
\hline 1. & Fruit shape & 1.578348 & 0.871665 & -1.24653 \\
\hline 2. & Coloring mode & -0.23281 & 0.691848 & 0.173174 \\
\hline 3. & Color type & 2.27393 & 2.051546 & -3.32845 \\
\hline 4. & Trichome presence & -0.54117 & 0.571291 & 0.467989 \\
\hline 5. & Hair length & -0.50498 & 0.545862 & 0.444604 \\
\hline 6. & Hair coloring & -0.36187 & 0.617713 & 0.489408 \\
\hline 7. & Position of attaching & 0.194314 & 0.461728 & 0.273855 \\
\hline 8. & Fruit surface sculpture & 2.058189 & 2.095991 & -0.0891 \\
\hline 9. & Fruit weight (mg) & -0.15868 & -0.01955 & 0.347091 \\
\hline 10. & Fruit wide (mm) & 0.125955 & 0.069094 & -0.25377 \\
\hline 11. & Fruit length (mm) & -0.18849 & 0.39183 & 0.305645 \\
\hline 12. & Section outline shape & 3.173544 & -3.89842 & -0.03563 \\
\hline 13. & Hull cells type & 0.443799 & 0.150657 & 1.117598 \\
\hline 14. & Aleurone cells shape & 0.12138 & 0.430593 & 1.567286 \\
\hline 15. & Aleurone cells orientation & 0.052685 & 0.232461 & 1.360735 \\
\hline 16. & Scutellum shape & 1.010615 & -1.21997 & 0.271807 \\
\hline 17. & Endosperm differentiation & -0.33176 & 0.452823 & 0.673013 \\
\hline 18. & Endosperm type & -0.33784 & 0.490211 & 0.698834 \\
\hline 19. & Section wide $(\mu \mathrm{m})$ & 0.296755 & -0.18378 & 0.934019 \\
\hline 20. & Section length $(\mu \mathrm{m})$ & 0.097103 & -0.48171 & 0.569057 \\
\hline 21. & Hull cells thickness $(\mu \mathrm{m})$ & -0.60307 & 0.690619 & 0.471997 \\
\hline 22. & Seed coat thickness $(\mu \mathrm{m})$ & 0.18211 & -0.36414 & 0.73636 \\
\hline 23. & Aleurone layer thickness $(\boldsymbol{\mu m})$ & 0.130375 & -0.39784 & 0.654374 \\
\hline 24. & Scutellum cells thickness $(\boldsymbol{\mu m})$ & -0.18054 & 1.09338 & 0.69615 \\
\hline 25. & Endosperm thickness $(\mu \mathrm{m})$ & 0.764397 & -0.05966 & 1.460169 \\
\hline 26. & Pollen class & -1.07075 & -0.2744 & -0.64054 \\
\hline 27. & Pollen size & -1.05331 & -0.44013 & -1.01514 \\
\hline 28. & Pollen shape & -0.92265 & -0.79847 & -0.89728 \\
\hline 29. & Pollen surface sculpture & -0.98552 & -0.54043 & $-\mathbf{0 . 8 4 1 4 8}$ \\
\hline 30. & Annulus thickness ( $\mu \mathrm{m})$ & -0.96595 & -0.83463 & -1.30983 \\
\hline 31. & Pore diameter $(\mu \mathrm{m})$ & -1.02089 & -0.78764 & -0.8329 \\
\hline 32. & Pollen wall thickness $(\mu \mathrm{m})$ & -1.00316 & -0.51699 & -0.97543 \\
\hline 33. & Sexine thickness $(\boldsymbol{\mu m})$ & -1.06557 & -0.39242 & -1.01249 \\
\hline 34. & Nexine thickness $(\boldsymbol{\mu m})$ & -0.97452 & -0.69914 & -1.23458 \\
\hline & Percentage per PCA & 16.54 & 4.34 & 3.13 \\
\hline
\end{tabular}

*PCA: Principal Component Analysis 
32. Aegilops kotshyi belongs to Triticeae. 3) Sub-clad of 33. Aegilops ventricosa, 34. Hordium murinum Subsp. Leporinum, 26. Lolium perenne, 15. Bromus scoparius, 13. Brachypodium distachym and 9. Avena fatua belonging to Triticeae, Poeae, Bromeae, Brachypodieae and Aveneae. 4) Sub-clad of species 29. Stipa capensis, 24. Dactylis glomerata, 30. Stipa lagascae, 14. Bromus rubens, 22. Echinochloa colona, 16. Coelachyrum bervifolium, 7. Schismus arabicus, 31. Stipa parviflora and 3. Aristida funiculata belonging to Stipeae, Poeae, Bromeae, Paniceae, Eragrostideae, Arundineae and Aristideae. 5) Sub-clad of species 27. Poa annua, 11. Polypogon maritimus, 19. Eragrostis minor, 10. Phalaris minor, 8. Avena barbata, 4. Aristida mutabilis, 21. Cenchrus ciliaris, 20. Leptochloa fusca and Aristida adscensionis within tribes: Poeae, Aveneae, Eragrostideae, Paniceae and Aristideae.

Several various monophyletic species which regarded as sister-groups are distinct within five subclads mentioned. Firstly, in the tree (Figure 1) Paniceae and Arundineae are a two-species sister-group to the rest whole cluster of the tree, on the other hand, Eragrostideae and Andropogoneae are another two-species sister-group within the second branch of the second major clad in the tree. Moreover, other species within Paniceae, Eragrostideae and Arundineae are separated through some different subclads; thus Andropogoneae alone can be conspicuously differentiated from other tribes by means of its characteristic features for the fruit morphology, fruit anatomy and pollen grains morphology. Secondly, different tribes consume sister-grouping within each of the five subclads distinguished. Tribe Poeae conform a monophyletic sister-group in subclad (1) in a cluster of Stipeae, Aveneae, Eragrostideae and Aristideae and in subclad (5) in a cluster of Stipeae, Eragrostideae, Aristideae, Bromeae, Paniceae and Arundineae. Therefore, Poeae is preferably separated from these tribes depending on its own marked pollen grains characters.

Moreover, the Triticeae shows a distinct variation that can aid the comparison of the relationships between Triticeae, Bromeae and Brachypodieae revealed by [16], where they suggested that the Brachypodieae is the sister group of the Triticeae while the Bromeae is the sister group of the Brachypodieae plus the Triticeae. Brachypodium is the sister-group of a clad including both Bromus and the Triticeae. While, [5] illustrated that the relationships between Bromus and the Triticeae is unresolved, so there is a possibility that the Triticeae is a non-monophyletic group. Meanwhile, in our results, the Triticeae is a monophyletic sister-group to the neighboring clad of Triticeae, Poeae, Bromeae, Brachypodieae and Aveneae (sub-clad 3). This clad which can be separated conspicuously through the Aveneae which is a monophyletic branch through Avena fatua, in addition to the separation of Poeae among tribes of subclads 1 and 5 . Thus the Triticeae, Bromeae and Brachypodieae are closely related as confirmed by their palynological similarity, in addition to the compatibility of the fruit morphological (Table 5) that enhanced the understanding of the degree of similarity between taxa of these tribes. The Stipeae is a sister-group of the sub-clad (4), with excluding tribes Poeae, Bromeae, Paniceae, Arundineae and Eragrostideae from this sub-clad, thus the Stipeae is separated from the Aristideae and also the similarity degree between them can be conducted to the characters of the fruit morphology and pollen grain morphology illustrated in Table 5. Therefore, the applied methods of UPGMA and PCA can be used to study the variation within the tribe and the tribes in the family to determine the relationships between genera and tribes. Our results revealed there is a much separation between tribes Andropogoneae, Arundineae, Aristideae, Stipeae, Poeae and Eragrostideae. However, tribes Triticeae, Bromeae and Brachypodieae showed much closer relationships. In addition to the consideration of those tribes Aveneae, Eragrostideae and Stipeae are the most heterogeneous tribes because the taxa of these tribes found to be interspersed with taxa from tribes Poeae, Paniceae and Aristideae.

\section{REFERENCES}

[1] W. D. Clayton and S. A. Renvoize, "Grasses of the World," Genera Graminum, Her Majesty's Stationary Office, London, 1986.

[2] H. P. Linder and P. J. Rudall, "The Evolutionary History of Poales," Annual Reviews in Ecology and Systematics, Vol. 36, No. 1, 2005, pp. 107-124. doi:10.1146/annurev.ecolsys.36.102403.135635

[3] Grass Phylogeny Working Group, "Phylogeny and Subfamilial Classification of the Grasses (Poaceae)," Annals of the Missouri Botanical Garden, Vol. 88, No. 3, 2001, pp. 373-457. doi: $10.2307 / 3298585$

[4] K. W. Hilu and K. Wright, "Systematics of Gramineae: A Cluster Analysis Study," Taxon, Vol. 31, No. 1, 1982, pp. 9-36. doi:10.2307/1220585

[5] K. W. Hilu and L. A. Alice, "A Phylogeny of Chloridoideae (Poaceae) Based on matK Sequences," Systematic Botany, Vol. 26, No. 2, 2001, pp. 386-405.

[6] O. Seberg and S. Frederiksen, "A Phylogenetic Analysis of the Monogenomic Triticeae (Poaceae) Based on Morphology," Botanical Journal of the Linnean Society, Vol. 136, No. 1, 2001, pp. 75-97. doi:10.1111/j.1095-8339.2001.tb00557.x

[7] C. L. McIntyre, "Variation in Isozyme Loci in Triticeae," Plant Systematics and Evolution, Vol. 160, No. 1-2, 1988, pp. 123-142. doi:10.1007/BF00936714

[8] J. V. Monte, C. L. McIntyre and J. P. Gustafson, "Analysis of Phylogenetic Relationships Using RFLPs," Theo- 
retical and Applied Genetics, Vol. 86, No. 5, 1993, pp. 649-655. doi:10.1007/BF00838722

[9] R. J. Mason-Gamer, E. A. Kellogg, "Chloroplast DNA Analysis of the Monogenomic Triticeae: Phylogenetic Implications and Genome-Specific Markers," In: J. J. Jauhar, Ed., Methods of genome analysis in plants. Boca Raton, CRC Press, Florida, 1996, pp. 301-325.

[10] E. A. Kellogg and R. Apple, "Intraspecific and Interspecific Variation in 5S RNA Genes are Decoupled in Diploid Wheat Relatives," Genetics, Vol. 140, No. 1, 1995, pp. 325-343.

[11] W. K. Taia, "Modern Trends in Plant Taxonomy," Asian Journal of Plant Sciences, Vol. 4, No. 2, pp. 2005, 184202.

[12] L. R. Parenti, "A Phylogenetic Analysis of the Land Plants," Biological Journal of the Linnean Society, Vol. 13, No. 3, 1980, pp. 225-242. doi:10.1111/j.1095-8312.1980.tb00084.x

[13] B. R. Baum, "A Phylogenetic Analysis of the Tribe Triticeae (Poaceae) Based on Morphological Characters of the Genera," Canadian Journal of Botany, Vol. 61, No. 2, 1983, pp. 518-535.
[14] J. Felsenstein, "Alternative Methods of Phylogenetic Inference and Their Interrelationship," Systematic Zoology, Vol. 28, No. 1, 1979, pp. 49-62. doi: $10.2307 / 2412998$

[15] K. Bremer, "Gondwanan Evolution of the Grass Alliance of Families (Poales)," Evolution, Vol. 56, No. 7, 2002, pp. 1374-1387.

[16] R. R. Sokal and C. D. Michener, "A Statistical Method for Evaluating Systematic Relationships," University of Kansas Scientific Bulletin, Vol. 28, 1958, pp. 1409-1438.

[17] A. K. Osman, "Numerical Taxonomic Study of Some Tribes of Compositae (Subfamily Asteroideae) from Egypt," Pakistan Journal of Botany, Vol. 43, No. 1, 2010, pp. 171-180.

[18] O. Seberg, S. Frederiksen, C. Baden and I. Linde-Laursen, "Peridictyon, a New Genus from the Balkan Peninsula, and Its Relationship with Festucopsis (Poaceae),"Willdenowia, Vol. 21, No. 1-2, 1991, 87-104.

[19] S. Frederiksen and O. Seberg, "Phylogenetic Analysis of the Triticeae (Poeae)," Hereditas, Vol. 116, No. 1-2, 1992, pp. 15-19. doi:10.1111/j.1601-5223.1992.tb00198.x 